\title{
A CLASS OF LATTICE ORDERED ALGEBRAS ${ }^{1}$
}

\author{
BY CASPER GOFFMAN
}

\author{
Communicated by Garrett Birkhoff, April 1, 1958
}

1. Our purpose is to characterize those lattice ordered algebras which may be represented as algebras of Carathéodory functions. This work is, accordingly, a sequel to [1] where the same problem was considered for lattice ordered groups. The rings considered here are more restrictive than those of Birkhoff and Pierce in [2], where an " $F$-ring" is shown to be isomorphic to a subring of the direct union of totally ordered rings (but the multiplication in [2] is not necessarily that which may be expected for functions; indeed, all products may be zero. In our case, the axioms compel the algebra multiplication to conform to that of the Carathéodory functions). Brainerd [3] has considered a class of algebras which have function space representations, but his emphasis is different from ours.

2. In this section, we define a Carathéodory algebra. Let $B$ be a relatively complemented distributive lattice. Let $E$ be the set of forms $f=a_{1} \alpha_{1}+\cdots+a_{n} \alpha_{n}$, where $\alpha_{i} \in B, a_{i}$ real, $i=1, \cdots, n$. With $f \geqq 0$ if $a_{i} \geqq 0$ for all $i$, and addition and multiplication defined by $f+g=\sum_{i=1}^{n} \sum_{j=1}^{m}\left(a_{i}+b_{j}\right)\left(\alpha_{i} \cap \beta_{j}\right)+\sum_{i=1}^{n} a_{i}\left(\alpha_{i}-\bigcup_{j=1}^{m} \beta_{j}\right)$ $+\sum_{j=1}^{m} b_{j}\left(\beta_{j}-\bigcup_{i=1}^{n} \alpha_{i}\right)$ and $f g=\sum_{i=1}^{n} \sum_{j=1}^{m} a_{i} b_{j}\left(\alpha_{i} \cap \beta_{j}\right)$ where $f=\sum_{i=1}^{n} a_{i} \alpha_{i}$ and $g=\sum_{j=1}^{m} b_{j} \beta_{j}, E$ is a lattice ordered algebra, which we call the algebra of elementary Carathéodory functions. Let $\bar{E}$ be the conditional completion of $E . \bar{E}$ is the set of bounded Caratheodory functions. In order to define the general Carathéodory function, we need the notion of carrier. In a lattice ordered group, for every $x \geqq 0$, $y \geqq 0$, we say $x \sim y$ if $x \cap z=0$ when and only when $y \cap z=0$. The equivalence classes obtained in this way are called carriers (filets by Jaffard [4]) and form a relatively complemented distributive lattice. The equivalence class to which $x$ belongs is called the carrier of $x$. In $\bar{E}$, consider pairwise disjoint sequences $\left\{f_{n}\right\}$ whose carriers have an upper bound, and consider the formal sums $\sum f_{n}$. With order, addition, and multiplication defined appropriately, these formal sums constitute a lattice ordered algebra - the Carathéodory algebra $C$ generated by $B$. (For details on related matters see $[5 ; 6]$ and $[1]$.)

3. Let $R$ be an archimedean lattice ordered algebra. Then $R$ is a lattice with positive cone $P$ such that $x, y \in P, a \geqq 0$ real, implies

${ }^{1}$ Supported by National Science Foundation grant no. NSF G-2267 on ordered systems. 
$x+y, x y, a x \in P$, and if $x, y \in P, y>0$, implies there is a real $a \geqq 0$ with $x-a y \notin P$. We say that $R$ is totally complete if

(a) $R$ is conditionally complete.

(b) every sequence of pair-wise disjoint elements in $P$, whose sequence of carriers has an upper bound, itself has an upper bound; hence, a least upper bound.

In addition to the archimedean hypothesis, the following condition is important for us.

A. If $x, y, z$ are in $P$ (i.e., $x \geqq 0, y \geqq 0, z \geqq 0)$ then $(x y) \cap z=0$ if and only if $x \cap y \cap z=0$.

It is not hard to see that the Caratheodory algebra $C$ is totally complete and satisfies $A$.

4. Before considering the main problem, we point out that for every totally complete vector lattice $R$, multiplication may be defined so that $R$ is an algebra satisfying $A$. We outline the procedure.

Let $\left[u_{\alpha}\right]$ be a generalized weak unit [1] in $R$. Then, for every carrier $\alpha$, there is a unique $u_{\alpha}$ with carrier $\alpha$, and for every $\alpha, \beta$ we have $u_{\alpha} \cap u_{\beta}=u_{\alpha \cap \beta}$ and $u_{\alpha} \cup u_{\beta}=u_{\alpha \cup \beta}$. For every $x>0$ there is, by the total completeness of $R$, a pairwise disjoint sequence $\left\{u_{\alpha_{n}}\right\}$ and a sequence $\left\{a_{n}\right\}$ of positive reals, such that $\sup a_{n} u_{\alpha_{n}} \geqq x$. For every $x>0, y>0$ let $u_{\alpha_{n}}, a_{n}$ be as above relative to $x$ and $v_{\beta_{n}}, b_{n}$ as above relative to $y$. Let $\xi=\sup \left(a_{n} u_{\alpha_{n}}\right)\left(b_{m} v_{\beta_{m}}\right)$. Then define $x y=\inf \xi$ for all $\xi$ obtained in this way. For any $x, y \in R$, define $x y=x^{+} y^{+}+x^{-} y^{-}-x^{+} y^{-}-x^{-} y^{+}$. It can then be shown that $R$ is an algebra satisfying $A$. Moreover, if $R$ has a weak unit, the resulting algebra has an identity.

5. We now let $R$ be a totally complete lattice ordered algebra, satisfying $A$.

Lemma 1. If $x \geqq 0, y \geqq 0$ then $x y=0$ if and only if $x \cap y=0$.

LEMma 2. If $x \geqq 0$ then $x$ and $x^{2}$ have the same carrier.

Proof. $x \cap y=0$ implies $x \cap x \cap y=0$ implies $x^{2} \cap y=0$. Conversely, $x^{2} \cap y=0$ implies $x \cap x \cap y=0$ implies $x \cap y=0$. More generally,

Lemma $2 '$. If $x, y \geqq 0$ have the same carrier, then $x y$ also has this carrier.

COROllary 1. Every carrier is a semi-ring.

Since $R$ is conditionally complete, for every $x, y \in R$, the projection $y_{x}$ of $x$ on $y$ is defined.

Lemma 3. $x y=x y_{x}$. 
The next lemma is important for us.

Lemma 4. If $x>0$ there is $y>0$ with $y x \geqq x$ and $z>0$ with $z x \leqq x$.

We outline the proof. From Lemma 2, the supremum of the carriers $\alpha_{n}$ of $w_{n}=\left(n x^{2}-x\right)^{+}$is the carrier of $x$. Let $\beta_{n}=\alpha_{n}-\alpha_{n-1}$ and let $z_{n}$ have carrier $\beta_{n}$. If $y_{n}=(n x)_{z_{n}}$, the $y_{n}$ are pair-wise disjoint. By the total completeness of $R$, sup $y_{n}=y$ exists. Then $y x \geqq x$. The proof of the second part is similar.

Definition. For every $x \geqq 0, u(x)=\inf [y \mid y x \geqq x]$ and $\bar{u}(x)$ $=\sup [y \mid y x \leqq x]$.

Lemma 5. For every $x \geqq 0, x=u(x) x=\bar{u}(x) x$.

Proof. $u(x) x \geqq x$. If $u(x) x>x$ there is $z>0$ with $z x<u(x) x-x$, whereby $(u(x)-z) x>x$, which is impossible.

Lemma 6. $[u(x)]^{2}=u(x)$ and $[\bar{u}(x)]^{2}=\bar{u}(x)$.

Proof. $[u(x)]^{2} x=u(x)[u(x) x]=u(x) x=x$ so that $[u(x)]^{2} \geqq u(x)$. Similarly, $[\bar{u}(x)]^{2} \leqq \bar{u}(x)$. But $\bar{u}(x) x=x$ implies $\bar{u}(x) \geqq u(x)$. However, $\bar{u}(x) \leqq u(x)$.

Corollary 2. $u(x)=\bar{u}(x)$.

Lemma 7. The carriers of $x$ and $u(x)$ are the same.

Proof. By condition A.

LEMMA 8. If $x$ and $y$ have the same carrier then $u(x)=u(y)$.

PRoof. If $0<x<z<y$ and $x^{2}=x, y^{2}=y$ then $z^{2}=z$. Let $\alpha$ be the carrier of $x$ and $y$. If $u(x) \neq u(y)$, there is $\beta<\alpha$ and $k<1$ such that, say, $k(u(x))_{w}<(u(y))_{w}$, where $w$ has $\beta$ as carrier. But then $\left[k(u(x))_{w}\right]^{2}$ $=k(u(x))_{w}$ and $k(u(x))_{w}=(u(x))_{w}$. This is impossible.

Thus there is a one-one correspondence $\alpha \rightarrow u_{\alpha}$ between the carriers and idempotents. There is a unique left identity for every carrier relative to the carrier; there is also a unique right identity.

Lemma 9. For every $\alpha$, the associated right and left identities are equal.

Proof. Both are idempotents. The proof is then as for Lemma 8. We summarize:

TheORem 1. A totally complete lattice ordered algebra $R$ satisfying $A$ has a unique idempotent $u_{\alpha}$ with carrier $\alpha$, for every $\alpha$. The idempotent $u_{\alpha}$ is an identity (left and right) for all $x \in R$ whose carrier is $\leqq \alpha$.

Corollary 3. The family $\left[u_{\alpha}\right]$ is a generalized weak unit in $R$. 
Proceeding as in [1], the algebra $R$ can be reconstructed from the $u_{\alpha}$ and a one-one correspondence obtained between the elements of $R$ and those of the space $C$ of Caratheodory functions generated by the relatively complemented distributive lattice $B$ of carriers in $R$. In this correspondence, each element $a_{1} u_{\alpha_{1}}+\cdots+a_{n} u_{\alpha_{n}} \in R$ is mated with the element $a_{1} \alpha_{1}+\cdots+a_{n} \alpha_{n} \in C$. It is then a routine matter to check that this correspondence preserves order, addition, and multiplication. We thus have:

Theorem 2. A lattice ordered algebra is isomorphic with the algebra $C$ of Carathéodory functions generated by a relatively complemented distributive lattice if and only if it is totally complete and satisfies $A$; i.e., for $x, y, z \geqq 0,(x y) \cap z=0$ if and only if $x \cap y \cap z=0$.

The following conditions are closely related to $A$.

$\mathrm{A}^{\prime}$. If $x, y \geqq 0$, then $x y=0$ if and only if $x \cap y=0$.

$\mathrm{A}^{\prime \prime} . R$ is an $F$-ring with no nonzero nilpotents.

Indeed, M. Henriksen has shown (oral communication) that conditions $A, A^{\prime}, A^{\prime \prime}$ are equivalent. Using this fact, and a completion theorem of Nakano [7] we obtain:

COROLlary 4. An archimedean lattice ordered algebra which satisfies $A$, and is such that inf $S=0$ and $x \geqq 0$ implies inf $x S=0$, is isomorphic with a subalgebra of a Caratheodory algebra.

We also obtain the following fact, which was proved in a different way for $F$-rings by Birkhoff and Pierce.

Corollary 5. An archimedean lattice ordered algebra which satisfies $A$ has commutative multiplication.

\section{REFERENCES}

1. C. Goffman, Remarks on lattice ordered groups and vector lattices, I. The Carathéodory functions, Trans. Amer. Math. Soc. vol. 88 (1958) pp. 107-120.

2. G. Birkhoff and R. S. Pierce, Lattice ordered rings, An. Acad. Brasil Ci. vol. 28 (1956) pp. 41-69.

3. B. Brainerd, On a class of lattice ordered rings, Proc. Amer. Math. Soc. vol. 8 (1957) pp. 673-683.

4. P. Jaffard, Contribution a l'etude des groupes ordonnés, J. Math. Pures Appl. vol. 32 (1953) pp. 203-280.

5. C. Carathéodory, Entwurf fïr eine algebraisierung des Integralbegriffs, Bayer Akad. Wiss. Math.-Nat. K1. Abh. (1938) pp. 28-67.

6. D. A. Kappos, Ein Beitrag zur Carathèodoryschen Definition der Ortsfunktionen, in Booleschen Algebren, Math. Z. vol. 51 (1949) pp. 616-634.

7. H. Nakano, Modern spectral theory, Tokyo, 1950, pp. 148-154.

PuRdue University aNd UNIVERSITY OF OKLAHOMa 